\title{
CoCo trial: Color-coded blood pressure Control, a randomized controlled study
}

\author{
This article was published in the following Dove Press journal: \\ Patient Preference and Adherence \\ 16 October 2014 \\ Number of times this article has been viewed
}

\section{Corinne Chmiel \\ Oliver Senn \\ Thomas Rosemann \\ Valerio Del Prete \\ Claudia Steurer-Stey \\ Institute of General Practice and Health Services Research, University \\ of Zurich, Zürich, Switzerland}

\begin{abstract}
Background: Inadequate blood pressure (BP) control is a frequent challenge in general practice. The objective of this study was to determine whether a color-coded BP booklet using a traffic light scheme (red, > $180 \mathrm{mmHg}$ systolic BP and/or $>110 \mathrm{mmHg}$ diastolic BP; yellow, $>140-180 \mathrm{mmHg}$ systolic BP or $>90-110 \mathrm{mmHg}$ diastolic BP; green, $\leq 140 \mathrm{mmHg}$ systolic BP and $\leq 90 \mathrm{mmHg}$ diastolic BP) improves BP control and adherence with home BP measurement.
\end{abstract}

Methods: In this two-group, randomized controlled trial, general practitioners recruited adult patients with a BP $>140 \mathrm{mmHg}$ systolic and/or $>90 \mathrm{mmHg}$ diastolic. Patients in the control group received a standard BP booklet and the intervention group used a color-coded booklet for daily home BP measurement. The main outcomes were changes in BP, BP control (treatment goal $<140 / 90 \mathrm{mmHg}$ ), and adherence with home BP measurement after 6 months.

Results: One hundred and twenty-one of 137 included patients qualified for analysis. After 6 months, a significant decrease in systolic and diastolic BP was achieved in both groups, with no significant difference between the groups $(16.1 / 7.9 \mathrm{mmHg}$ in the intervention group versus $13.1 / 8.6 \mathrm{mmHg}$ in the control group, $P=0.3 / 0.7$ ). BP control (treatment target $<140 / 90 \mathrm{mmHg}$ ) was achieved significantly more often in the intervention group ( $43 \%$ versus $25 \% ; P=0.037$; number needed to treat of 5). Adherence with home BP measurement overall was high, with a trend in favor of the intervention group $(98.6 \%$ versus $96.2 \% ; P=0.1)$

Conclusion: Color-coded BP self-monitoring significantly improved BP control (number needed to treat of 5 , meaning that every fifth patient utilizing color-coded self-monitoring achieved better BP control after 6 months), but no significant between-group difference was observed in BP change. A markedly higher percentage of patients achieved BP values in the normal range. This simple, inexpensive approach of color-coded BP self-monitoring is userfriendly and applicable in primary care, and should be implemented in the care of patients with arterial hypertension.

Keywords: primary care, home blood pressure measurement, hypertension, high blood pressure, general practitioner, number needed to treat

\section{Introduction}

Arterial hypertension ( $\mathrm{AH})$ is the most common chronic disease among Swiss adults apart from dyslipidemia and diabetes mellitus. ${ }^{1}$ Worldwide, the prevalence of AH among adults is estimated to be $26 \%$, representing a major risk factor for cardiovascular morbidity and mortality. ${ }^{2,3}$ Even though effective medication and guidelines for the treatment and management of AH exist, ${ }^{4}$ the control of high blood pressure (BP) in the community is far from optimum. ${ }^{5}$ In order to reach treatment goals in a chronic condition, adherence with treatment and patient collaboration are essential. To successfully cope with and manage a chronic disease in the long term it is necessary to acquire skills and to change behavior. Home BP measurement (HBPM) is an important skill for patients to monitor their disease and has been consistently recommended in hypertension guidelines. ${ }^{6,7}$ Yet
Institute of General Practice and Health Services Research, University of Zurich Pestalozzistrasse 24, 809I Zürich, Switzerland

Tel +4I 0442559855

Fax +4I 0442559097

Email corinne.chmiel@usz.ch 
low adherence with self-monitoring and treatment recommendations remains problematic in daily practice. ${ }^{8}$ While a vast amount of data exist regarding $\mathrm{AH}$ and adherence with treatment, ${ }^{9-11}$ relatively few studies and reviews have explored how adherence and patient self-monitoring ability can be supported and improved, and the conclusions of these studies are not consistent. ${ }^{12-17}$ Recommendations for research on interventions to improve adherence encourage development of interventions that are simple and easily applicable to the clinical setting. ${ }^{18}$ The aim of the Color-coded blood pressure Control (CoCo) trial was therefore to analyze the effect of a simple, practical tool, ie, a color-coded BP booklet with a traffic light scheme for self-monitoring, on BP control and adherence with self-measurements.

\section{Materials and methods}

CoCo is a two-group, randomized controlled trial designed to analyze the effect of a color-coded versus usual HBPM booklet on adherence with self-monitoring and BP control. The detailed study protocol and the baseline characteristics of the participating patients and GPs have been previously published elsewhere. ${ }^{19,20}$ The trial is registered under ClinicalTrials.gov ID NCT01013467.

\section{Sample size}

Case number calculation and power analysis were carried out on the basis of the expected effect for systolic BP. With an alpha of 0.05 and a power of 0.9 (one-sided test), this means that 138 patients were required in order to be able to show a clinically relevant BP difference of $7 \mathrm{mmHg}$ systolic with a standard deviation (SD) of $14 \mathrm{mmHg}$ between the groups. Assuming a dropout rate of $20 \%$, this meant that at least
166 patients were required. Since the dropout rate in our population was much lower, we almost managed to reach the predefined sample size of 138 patients.

\section{Recruiting and inclusion criteria}

General practitioners (GPs) from the greater area of Zurich and St Gallen were invited to consecutively recruit patients not or insufficiently treated for AH. Inclusion criteria were: age $>18$ years; $\mathrm{BP}>140 \mathrm{mmHg}$ systolic and/or $>90 \mathrm{mmHg}$ diastolic, where the second practice measurement qualified for inclusion; no or unchanged $\mathrm{AH}$ treatment for one month before inclusion; patient able to perform HBPM; and written informed consent. Exclusion criteria were: $\mathrm{BP}>180 \mathrm{mmHg}$ systolic and/or $>110 \mathrm{mmHg}$ diastolic (due to safety/risk considerations); serious general or psychological illness, such as malignant tumors, serious depression, or evidence of dementia; and insufficient knowledge of the German language for instruction on BP recordings with a booklet.

\section{Randomization}

Randomization took place at the patient level and was performed by the study center. We drew up a randomization list by computer (ralloc command of Stata software for Windows, version 9). Envelopes containing the patient's number and corresponding booklet were given to the GPs in numerical order and distributed by the GP to the patient in order of appearance and inclusion.

\section{Measurements}

Clinical and demographic data upon inclusion and at follow-up were collected by the GPs (Tables 1 and 2).

Table I Patient characteristics at inclusion according to randomization

\begin{tabular}{lll}
\hline Characteristic & Intervention group & Control group \\
\hline Number & 65 & 56 \\
Male (\%) & 53.9 & 44.6 \\
Age (years) & $61.5(I 3.1)$ & $62.0(12.6)$ \\
BMI (kg/m²) & $28.2(4.3)$ & $28.8(5.4)$ \\
Smokers (\%) & 21.5 & 23.2 \\
Ex-smokers (\%) & 29.2 & 28.6 \\
Education (years) & $11.4(4.4)$ & $10.7(4.1)$ \\
Number of AH drugs & $1.3(0.9)$ & $1.6(1.0)$ \\
No AH medication (\%) & 9.2 & 12.5 \\
HBPM (\%) & 61.5 & 64.3 \\
Systolic BP (mmHg) & $157.5(15.3)$ & $159.5(13.2)$ \\
Diastolic BP (mmHg) & $91.8(7.6)$ & $92.8(9.6)$ \\
Pulse rate per minute & $73.7(10.3)$ & $75.1(I I .7)$ \\
\hline
\end{tabular}

Note: Continuous variables are indicated as the mean and standard deviation.

Abbreviations: AH, arterial hypertension; BMI, body mass index; BP, blood pressure; HBPM, home blood pressure monitoring. 
Table 2 Follow-up measurements after 6 months according to randomization

\begin{tabular}{lll}
\hline Criteria & Intervention group & Control group \\
\hline Systolic BP $(\mathrm{mmHg})$ & $141.4(13.0)$ & $146.4(17.9)$ \\
Diastolic BP $(\mathrm{mmHg})$ & $83.8(9.8)$ & $84.2(\mathrm{II.7})$ \\
BP control $(\%)$ & $43.1^{\circ}$ & 25.0 \\
Change of systolic BP $(\mathrm{mmHg})$ baseline vs follow-up & $-16.1(16.3)$ & $-13.1(16.1)$ \\
Change of diastolic BP $(\mathrm{mmHg})$ baseline vs follow-up & $-7.9(9.4)$ & $-8.6(10.4)$ \\
\hline
\end{tabular}

Notes: Continuous variables are indicated as the mean and standard deviation. ${ }^{*} \mathrm{BP}<140 / 90 \mathrm{mmHg}$; ${ }^{\circ} \mathrm{P}<0.05$ between groups.

Abbreviations: BP, blood pressure; vs, versus.

Patients in the intervention group received the color-coded booklet (Figure 1A), with a scheme divided into three measuring zones (green, yellow, red) following a traffic light scheme. BP measurements within the target values of up to $140 \mathrm{mmHg}$ systolic and $90 \mathrm{mmHg}$ diastolic were entered into the green area. BP measurements above $140 \mathrm{mmHg}$ but below $180 \mathrm{mmHg}$ systolic and/or above $90 \mathrm{mmHg}$ but below $110 \mathrm{mmHg}$ diastolic were entered into the yellow area, and measurements above $180 \mathrm{mmHg}$ systolic and above $110 \mathrm{mmHg}$ diastolic in the red area. The colored areas had the goal to improve patients' understanding and interpretation of BP control by visualizing target and elevated values. Pulse rate was also recorded but without any designated color coding. Patients in the control group used the usual standard booklet (Figure 1B) without color coding. In both booklets, the exact time and date of readings were recorded.

Automatic electronic oscillometric BP measurement devices (MioStar Cardioplus 500) for measurement by GPs as well as for HBPM were provided to the GP by the Institute of General Practice in order to minimize data

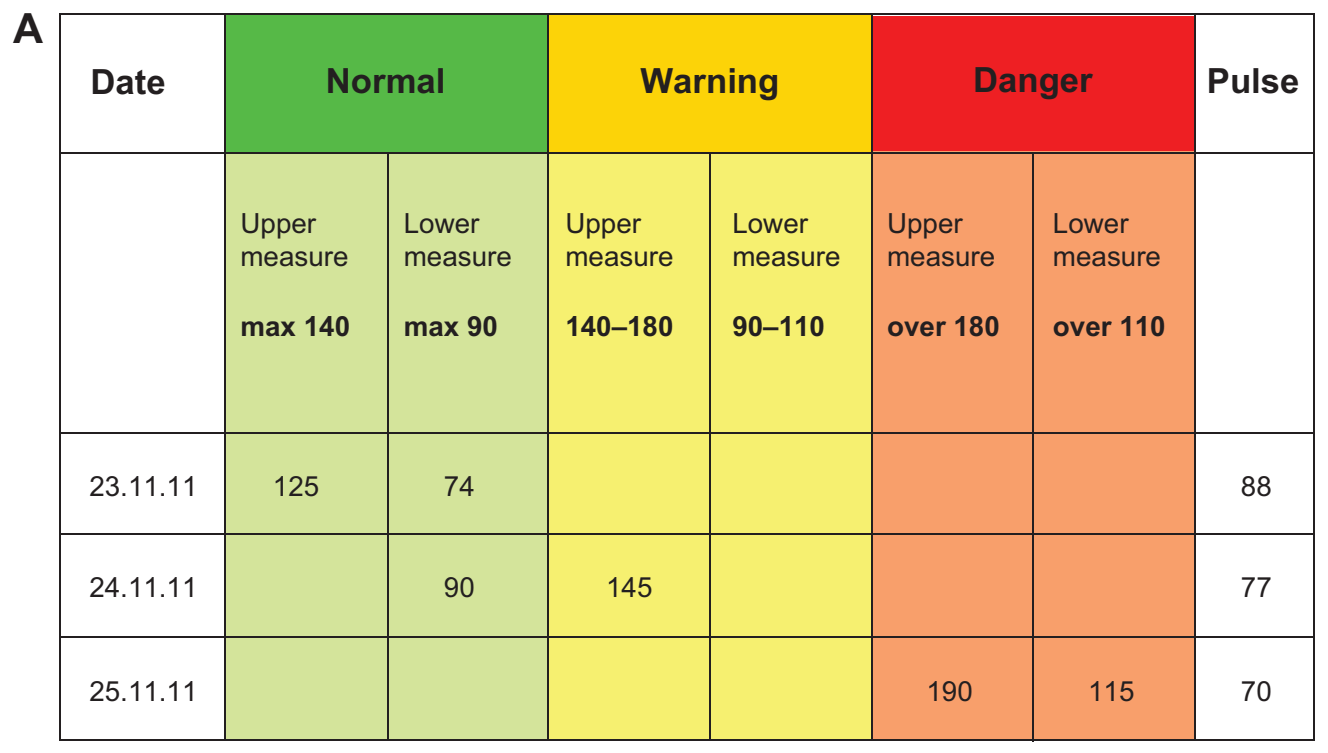

\begin{tabular}{|c|c|c|c|}
\hline Date & Upper measure & Lower measure & Pulse \\
\hline 23.11 .11 & 125 & 74 & 88 \\
\hline 24.11 .11 & 145 & 90 & 77 \\
\hline 25.11 .11 & 190 & 115 & 70 \\
\hline
\end{tabular}

Figure I (A) Color-coded and (B) standard blood pressure booklet. 
variability through technical differences between devices. The device is validated for accuracy of measurement according to EU-norm EN1060 and is easy to handle. In order to reflect a real life situation, this specific model was chosen after previous discussion with GPs on which device is mainly used by Swiss patients for HBPM. GPs and patients were instructed to perform BP measurements according to the guidelines. ${ }^{7,21,22}$ GPs were instructed to choose an appropriate cuff size according to the patient's upper arm circumference. The patients were provided with the device and appropriate cuff by the GP at the time of inclusion in the study. For baseline BP measurement at the GP office, two consecutive BP readings were performed in the seated position after a resting period of at least 5 minutes. To rule out differences in BP, measurements were performed on both arms at baseline. Each subsequent measurement in the GP office involved two BP measurements in each case in the left arm if the initial measurement did not differ between the two arms by more than $5 \mathrm{mmHg}$. If the measurement differed by more than $5 \mathrm{mmHg}$ at baseline, BP was measured at the arm with the higher reading subsequently. All the readings were recorded, but for inclusion as well as for analysis, the second measurement was used. Patients were trained by their GPs in correct BP measurement and recording. They also received detailed written and illustrated instruction on how to perform HBPM. They were instructed to perform BP measurements at least every morning, before medication intake, in a seated position, after a resting period of at least 5 minutes, on the left upper arm with the lower arm resting on the table. Missing measures were not to be supplemented but entered as a dash into the BP booklet. More than one measurement per day was allowed; the additional data were not considered for analysis concerning adherence to HBPM. Adherence to HBPM was defined as the percentage of days with an entry of BP measurement during the period of observation.

The study period was 6 months for both groups. After 3 and 6 months, a follow-up by the GP took place, where clinical parameters and medication changes were recorded.

The primary endpoint was the difference in systolic and diastolic BP readings measured at the practice between baseline and after 6 months of follow-up. Secondary endpoints were the number of patients who achieved BP control $<140 / 90 \mathrm{mmHg}$ at the practice measurement and adherence, ie, the absolute number of BP measurements (entries in the BP record booklet) by the patients was assessed as correlative of adherence during the period of observation.

\section{Data processing and ethical approval}

The anonymous data forms were sent to the project leaders at the Institute of General Practice and the data were entered into an Excel database. All patients gave written informed consent. The study protocol was approved by the ethics committee of Zurich (Kantonale Ethikkommission, Zürich, Switzerland, EK-1738).

\section{Statistical analysis}

Continuous variables were tested for normality distribution by the Shapiro-Wilk test and presented accordingly as the median and interquartile range or as the mean and SD, and categorical data are presented as frequencies. For categorical data, the $\chi^{2}$ test was used to assess treatment group comparisons, for continuous variables, comparisons between treatment groups were performed via unpaired $t$-tests. All analyses were performed in accordance with the "intentionto-treat" approach and the latest available measurement was used (last observation carried forward). For further analysis of the primary and secondary endpoints, we controlled for BP levels at baseline and used multilevel regression analysis with the GP as the cluster level, thus taking into account that patient observations are not independent, ie, observations in one cluster tend to be more similar to each other than to individuals in the rest of the sample. The amount of clustering (ie, the variation that is explained by the GP level) was assessed by calculating the intraclass correlation coefficient (ICC) ranging from $0 \%$ (ie, no evidence for clustering) to $100 \%$ (ie, all the variation in BP difference is explained at the GP level). The adjusted effect of the booklet on HBPM values (weekly averages over 26 weeks) controlled for time was analyzed using a multilevel repeated-measures design with the patient as the level of clustering. A two-sided alpha of 0.05 was set as the level of significance for all comparisons. All analyses were calculated using the Stata statistical package version 11.2 (Stata Incorporation, College Station, TX, USA).

\section{Results \\ Study population}

The number of participants during the study is depicted in the study flow chart (Figure 2). Between October 2009 and November 2011, 137 patients were assessed for eligibility by 30 GPs. Seventy patients were allocated to the intervention group, of whom 65 were eligible for analysis, and 67 patients were randomized to the control group, of whom 56 were eligible for analysis. Randomization was successful, showing no 


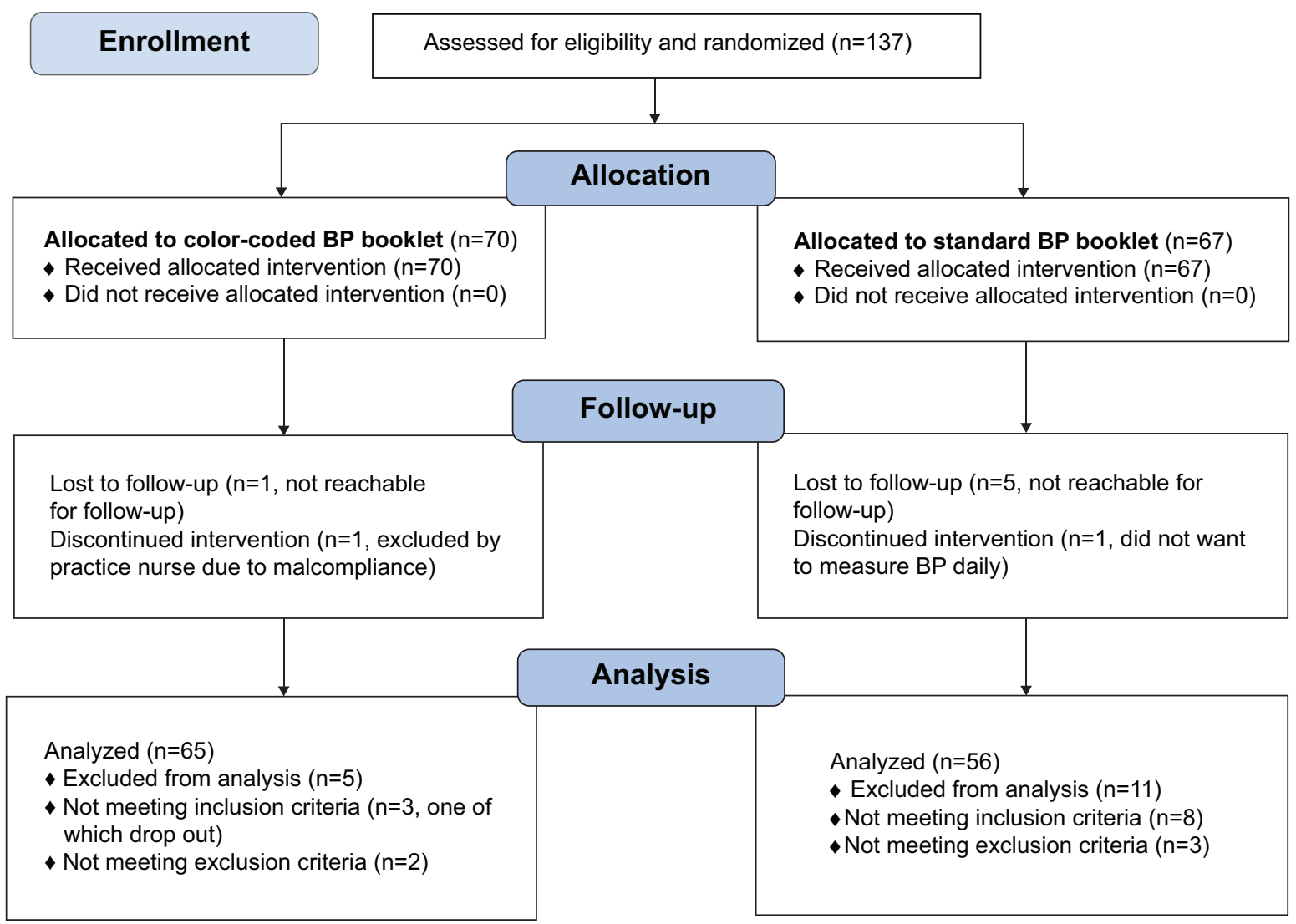

Figure 2 Study flow chart.

Abbreviation: $\mathrm{BP}$, blood pressure.

significant difference in any baseline characteristics between the control group and intervention group (Table 1).

\section{Primary endpoint: decrease of systolic and diastolic BP after 6 months}

Systolic and diastolic BP measurements performed by the GP showed a significant decrease after 6 months compared with the baseline measurements in both groups, with no significant difference in systolic and diastolic BP between the groups (Table 2). Mean (SD) systolic BP at baseline was $157.5 \pm 15.3 \mathrm{mmHg}$ in the intervention group and $159.5 \pm 13.2 \mathrm{mmHg}$ in the control group, and mean diastolic BP was $91.8 \pm 7.6 \mathrm{mmHg}$ in the intervention group and $92.8 \pm 9.6 \mathrm{mmHg}$ in the control group. Mean (SD) systolic BP at follow-up was $141.4 \pm 13.0 \mathrm{mmHg}$ in the intervention group and $146.4 \pm 17.9 \mathrm{mmHg}$ in the control group, and mean diastolic BP was $83.8 \pm 9.8 \mathrm{mmHg}$ in the intervention group and $84.2 \pm 11.7 \mathrm{mmHg}$ in the control group. The mean decrease in systolic BP showed no benefit of the colorcoded booklet $(16.1 \pm 16.3 \mathrm{mmHg}$ in the intervention group compared to $13.1 \pm 16.1 \mathrm{mmHg}$ in the control group $[P=0.3]$ ).
The mean diastolic BP decrease was 7.9 $\pm 9.4 \mathrm{mmHg}$ in the intervention group and $8.6 \pm 10.4 \mathrm{mmHg}$ in the control group $(P=0.7)$. The $P$-values did not significantly change when additionally controlled for a GP clustering effect and BP at baseline ( $P=0.123$, ICC $6.9 \%$ for systolic BP; $P=0.848$, ICC $1.8 \%$ for diastolic $\mathrm{BP})$.

\section{Secondary endpoints}

Number achieving BP control and adherence to HBPM

BP control $(<140 / 90 \mathrm{mmHg})$ was achieved significantly more often in the intervention group than in the control group (43.1\% versus $25.0 \% ; P=0.037$, Table 2 ). The $P$-value did not change when additionally controlled for a GP clustering effect and BP at baseline ( $P=0.044$, ICC $16.3 \%)$. Adherence was defined as the percentage of days with an entry of a BP measurement in the booklet. Analysis of measurement frequencies recorded in the BP booklets revealed high adherence in both groups, with $98.6 \%$ completeness for BP measurement recordings in the intervention group and $96.2 \%$ in the control group, showing a trend in favor of the intervention group $(P=0.1)$. 


\section{Home BP measurements}

Analysis of weekly averages for HBPM values recorded in the booklets over 26 weeks showed a significant influence of color coding on systolic BP adjusted for time; using a color-coded booklet was independently associated with reduced systolic BP (regression coefficient $-4.26,95 \%$ confidence interval $[\mathrm{CI}]-7.85,-0.68 ; P=0.020$ ). No significant association with diastolic BP was found (regression coefficient $-1.03,95 \% \mathrm{CI}-4.22,2.15 ; P=0.53)$.

\section{Antihypertensive medication}

At the time of inclusion in the study, 108 patients (89.3\%) received AH treatment, of which $38.8 \%$ had monotherapy, $30.6 \%$ had dual therapy, and the remaining $19.8 \%$ took three or more AH drugs. No difference in distribution of AH therapy concerning number of $\mathrm{AH}$ drugs $(P=0.2831)$ was found between the two groups. The most commonly prescribed $\mathrm{AH}$ substances were diuretics (38.8\%) and angiotensin-converting enzyme (ACE) inhibitors (36.4\%), followed by angiotensin receptor type 2 (AT2) inhibitors (29.8\%). Concerning the distribution of AH substances, no difference between the groups existed, except for the control group being treated more often with $\mathrm{ACE}$ inhibitors when compared with the intervention group $(P=0.012)$.

At follow-up after 6 months, the proportion of patients without $\mathrm{AH}$ treatment had decreased from $10.7 \%$ to $3.5 \%$, with $21.2 \%$ receiving monotherapy, $30.1 \%$ dual therapy, and $45.1 \%$ three or more AH drugs. No difference in distribution concerning number of $\mathrm{AH}$ drugs was found between the two groups ( $P=0.1971$ ). Overall, in $63 \%$ of patients, the $\mathrm{AH}$ therapy was changed ( $68.1 \%$ of the control group and $59.7 \%$ of the intervention group). No difference existed in overall change of AH therapy between the groups ( $P=0.367$ for any alteration of dosage of any AH substance and/or amount of substances).

The most commonly prescribed AH drugs were diuretics (60.5\%) and AT2 inhibitors (56.1\%), followed by ACE inhibitors (47.3\%). Concerning the distribution of AH substances, no difference between the groups existed except for the intervention group being treated more often with AT2 inhibitors when compared with the control group $(P=0.007)$.

\section{Participating GPs}

Detailed characteristics on the 30 participating GPs have been reported previously. ${ }^{19}$ No difference could be found between the control group and the intervention group concerning the following GP characteristics: geographic distribution (urban, suburban, or rural), specialization (internal or general medicine), age, practice experience, time since completion of board examination, employment (part-time or full-time), and use of and time since implementation of electronic patient records. A significant difference between the groups was found with regard to the following GP characteristics: in the intervention group more GPs were male (39 versus 43 , $P=0.0336)$ and more worked in groups rather than singlehanded practices (36 versus $40, P=0.0113$ ).

\section{Discussion}

BP control, defined as the proportion of BP values within normal range, significantly improved in patients using the color-coded BP monitoring booklet. The absolute BP reduction did not differ between the groups and adherence with HBPM was high in both groups, with a trend in favor of the color-coded booklet. The effect of color-coded selfmonitoring on BP control can be translated in a number needed to treat of 5 , meaning that every fifth patient utilizing color-coded self-monitoring achieves better BP control after 6 months.

Various studies have shown that BP control is far below the optimum and treatment goals are achieved in currently half of the patients who are treated for $\mathrm{AH}^{23}$ Important elements associated with improved BP control are a rigorous stepped-care approach to $\mathrm{AH}$ drug treatment, organization of care with regular follow-up, and interventions and motivational strategies that aim to help patients achieve better medication use and BP management. ${ }^{24}$ HBPM is one of these supportive measures. Randomized controlled trials have shown that self-monitoring of BP results in better BP control. ${ }^{8,25,26}$ A recently published meta-analysis showed that HBMP alone was able to reduce BP over 6 months but not in the long term (more than 12 months). In patients with HBPM and additional support, the effect on long-term BP reduction was significantly improved. ${ }^{27}$

The rationale for color-coded BP monitoring was to motivate patients to self-monitor and to facilitate interpretation by visualized information with a traffic light system and thus trigger appropriate behavior. The concept behind such tools aiming to improve adherence and disease control is selfregulation. Self-regulation is meant to support the patient's perception and capability for decision-making and to help them to develop strategies and health behavior associated with better outcomes. ${ }^{28,29}$

To our knowledge, this is the first paper assessing the effect of color-coded self-monitoring without self-titration on BP control. Earlier studies comparing a patient-directed management strategy with office-based management in 
primary care used HBPM with an algorithm for upward and downward adjustments of drug therapy, but not a color traffic light algorithm. ${ }^{30}$ TASMINH2 (the Telemonitoring and Self-Management of Hypertension Trial) consisted of selfmonitoring of BP combined with telemonitoring by means of an automated modem device that was connected to a sphygmomanometer and self-titration of AH drugs according to a color traffic light system comparable with our scheme to code the BP readings. ${ }^{31}$ The primary endpoint was change in mean systolic BP. Mean systolic BP decreased by $12.9 \mathrm{mmHg}$ (95\% CI 10.4-15.5) from baseline to 6 months in the selfmanagement group and by $9.2 \mathrm{mmHg}(95 \% \mathrm{CI} 6.7-11.8)$ in the control group (difference between groups $3.7 \mathrm{mmHg}$, $95 \%$ CI 0.8-6.6; $P=0.013$ ) which is comparable with our results.

Several factors can explain why only a trend in benefit of the color-coded self-monitoring on BP reduction could be found in our study, although BP control as well as systolic values in HBPM showed a significant improvement in the group with the color-coded booklet. A Hawthorne and/or regression to the mean effect seems likely when considering the very significant systolic (approximately 13 and $16 \mathrm{mmHg}$ ) and diastolic (approximately $8 \mathrm{mmHg}$ ) BP decrease achieved in both groups, even though two thirds of patients already performed HBPM before inclusion in the study. The study was designed to estimate the effect over a period of 6 months. It is possible that the effect of color-coded monitoring might have increased after a longer time period. The most likely reason is that, unlike the TASMINH2 study, no action plan was tied to the color-coding. It has been shown for several chronic illnesses that self-management consisting of regular self-measurements and a written action plan for adjusting treatment is more effective than care without an action plan. ${ }^{31-36} \mathrm{We}$ almost reached the prespecified sample size, suggesting a lack of power or type 2 error as a possible explanation for not reaching significance concerning $\mathrm{BP}$ reduction, although significance for BP control as well as systolic BP in HBPM was achieved. Lack of blinding of participating GPs and patients to intervention might have biased the results as well.

In our study, patients were instructed to perform HBPM every morning before medication intake. No evening or daytime measurements were required by the protocol, as suggested in the guidelines. ${ }^{7,22}$ It was left to the discretion of the patient whether to perform additional measurements. It is clear that a whole day reflects BP control more in detail. We chose this simple protocol to reflect the real life situation, since it is not realistic to demand that patients perform an all-day protocol during a 6-month period. The study endpoint was change in BP measured at the GP's office and adherence to HBPM, not change in HBPM values; therefore, it was not necessary to analyze the whole day HBPM profile. The colorcoded BP booklet aimed at triggering behavioral change in the event of BP measurements beyond target, possibly resulting in altered patient-GP interaction and treatment adaptation. Hence it was not necessary to demand a more rigorous measuring protocol for HBPM. On the contrary, by requiring more HBPM measurements throughout the day, the intuitive effect of the color-coded booklet might have been anticipated by a rigorous study protocol. These considerations are reflected in the finding that many patients with HBPM values out of target performed additional measurements throughout the day because of their own motivation.

In our study, remarkable BP control as well as improved systolic BP in HBPM was achieved with color-coded selfmonitoring. The study did not assess the exact mechanisms of how the color-coded booklet managed to improve BP control when compared with the standard booklet. The detailed processes on the part of the patient and doctor as well as the interaction between the two remain a black box and offer interesting aspects to be studied further in daily practice. The booklet only offers a "surrogate" for many possible mechanisms leading to improved BP control. Since no difference in change of AH therapy between the groups was found in our study, this suggests that the effect of the color-coding can not only be reduced to medication change performed by the GP. The color-coding probably mediates lifestyle changes and better adherence to treatment in addition to alerting the GP to make medication changes when needed. The data reported by McManus et $\mathrm{al}^{31}$ showed that $70 \%$ of patients who selfmanaged their hypertension made at least one medication change. We can only assume that the better visualization of $\mathrm{BP}$ ranges in the color-coded booklet led to some form of self-management in patients and probably also better patientdoctor communication. The potential role of physicians in BP control and their behavioral change (beyond changes in prescriptions) was not addressed in our study. However, there is published work regarding the effect of self-monitoring itself demonstrating a positive effect on BP. ${ }^{16}$ In our study, we observed high levels of adherence with self-monitoring in both groups. A direct association between adherence to HBPM and BP control can therefore not be postulated. This puts emphasis on the color-coding with respect to BP control and might reflect that people need support in the interpretation of risk and that simple interpretation aids work and have the potential to induce healthy behavior. Even though there was a shift in use of AH substances from the baseline 
(more ACE inhibitors in the control group) to follow-up (more AT2 inhibitors in the intervention group), the overall escalation of therapy concerning number of $\mathrm{AH}$ drugs and substance distribution otherwise did not differ between the groups. Improved BP control therefore cannot be explained by an intensified step-up approach of AH drugs alone, and the color coding seems to have additional effects.

\section{Strengths and limitations}

There are several shortcomings to be mentioned. Along with the study information, the GPs received a list and instructions to register all patients they asked to participate and all patients who declined to participate. This list was unfortunately not completed by the majority of GPs, so it is unknown how many of the potentially eligible patients were included in the trial. The objective of the present study was to assess the effectiveness of a simple intervention aimed at improving BP control. Information that might possibly influence BP control, such as renal function, hyperaldosteronism, and BPinfluencing medications, such as non-steroidal antirheumatic drugs or systemic corticosteroids, was not collected. However, randomization was successful, showing no significant difference in baseline characteristics between the groups. It therefore seems justified to assume that comorbidities were also equally distributed. Statements concerning conformity of the prescribed AH treatments with the guidelines are not possible since comorbidities and drug intolerances were not recorded in the study data. In our study, adherence to treatment was not evaluated, but BP measurement was used as a surrogate for adherence. Nevertheless, we assume that patients willing to participate in the study, perform daily BP measurements, and enter them into a booklet are likely to comply with therapy. No HBPM measurement at baseline was available. We chose practice measures for primary and secondary outcomes due to their reproducibility. HBPM was only assessed after inclusion into the study. The BP measurement device was chosen according to its popularity in our study population and its practicality in usage. It is validated according to EU-norm EN1060, but it is uncertain whether it would pass the validation process of the European Society of Hypertension International Protocol. ${ }^{7,37}$ Since all participating GPs and patients received the same device, possible flaws in measurement accuracy were equalized through randomization of the study population. The device used in our study only offers a memory function for the last measured value. It was therefore not possible to check for the reliability of measurements recorded by patients in their booklets. The primary and secondary outcomes consisted of practice measurements performed by the GP that made the information on correctness of entries less relevant for the study outcome.

The strength of our study is that it offers a view on "real life" primary care patients, with uncontrolled AH in $90 \%$ of those on $\mathrm{AH}$ treatment at the time of inclusion into the study. Further, the low intensity and low complexity of the intervention makes it applicable for primary care, where $\mathrm{AH}$ is largely managed. GPs and patients are already used to and are using booklets for BP monitoring in daily practice. Therefore, our intervention adding only a color-coded traffic light scheme avoided the substantial extra effort which is often feasible under study conditions but not in practice over a longer time period.

\section{Perspectives}

While there is a strong evidence base for the benefits of $\mathrm{AH}$ drug therapy, there is less clear evidence for supplementary care, including self- monitoring of BP by patients. Our study results support color-coded self-monitoring using a traffic light scheme as a useful, simple, inexpensive, and applicable adjunct to care for people with AH. This is important in light of the fact that self-monitoring is now widely practiced. By means of emerging computerized monitoring, it will be possible to implement individualized color coding according to preferred BP targets. Color coding is an effective intervention that improves BP control; however, the relationship between control of $\mathrm{BP}$, self-monitoring, and self-management interventions needs further evaluation with quantitative as well as qualitative research. Additionally, evaluation of effect in larger, long-term, randomized controlled trials of cardiovascular morbidity and mortality is required.

\section{Conclusion}

Color-coded BP self-monitoring significantly improved BP control (number needed to treat of 5), but no significant between-group difference was observed in BP change. A remarkable higher percentage of patients achieved BP values in the normal range. This inexpensive, simple method of color-coded BP self-monitoring is easily applicable and feasible in primary care, and should be implemented in care for patients with $\mathrm{AH}$.

\section{Acknowledgments}

The study is funded by the Swiss Academy of Medical Sciences (approved on 30 April 2011) and Uniscientia Stiftung, Vaduz. BP measurement devices were provided 
at a reduced price by Melectronics, Zürich, Switzerland. The funding sources had no influence on study design; on the collection, analysis, and interpretation of data; on the writing of the manuscript; or on the decision to submit the manuscript for publication. We are very grateful to Kaba Dalla Lana, Anke Schickel, and Barbara Portmann for their administrative help and to the GPs who recruited and followed patients. We also thank Christian Häuptle from Kantonsspital, St Gallen, for support in recruiting participating GPs.

\section{Author contributions}

CC, OS, TR, and CSS developed the study protocol. CC was study investigator and wrote the drafts of the manuscript. CC, OS, and VDP performed statistical analysis and interpreted data. OS and CSS were study investigators and substantially contributed to and reviewed the drafts of the manuscript. CC and VDP were responsible for data collection. All authors revised drafts of the manuscript, read and approved the final manuscript.

\section{Disclosure}

CSS has an unrestricted grant from AstraZeneca Switzerland for chronic care and patient education. CSS works on advisory boards and gives talks for AstraZeneca, Novartis, Boehringer Ingelheim, and Merck Sharp and Dome. The other authors have no conflicts of interests to declare.

\section{References}

1. Swiss statistics. [webpage on the Internet]. Neuchâtel: Swiss Confederation; 2014. Available from http://www.bfs.admin.ch/bfs/portal/de/index/ themen/14/02/01/key/02.html. Accessed October 1, 2011.

2. Kearney PM, Whelton M, Reynolds K, Muntner P, Whelton PK, He J. Global burden of hypertension: analysis of worldwide data. Lancet. 2005;365(9455):217-223.

3. Hanninen MR, Niiranen TJ, Puukka PJ, Kesaniemi YA, Kahonen M, Jula AM. Target organ damage and masked hypertension in the general population: the Finn-Home study. J Hypertens. 2013;31(6): 1136-1143.

4. Swiss Society of Hypertension. 34es Journées de l'Hypertension Artérielle. [34th Hypertension Days]. Available from: http://swisshypertension.ch. Accessed August 24, 2014.

5. Kannel WB. Risk stratification in hypertension: new insights from the Framingham Study. Am J Hypertens. 2000;13(1 Pt 2):3S-10S.

6. Hypertension: The Clinical Management of Primary Hypertension in Adults. [webpage on the Internet]. London: National Institute for Health and Care Excellence; 2011. Available from http://www.nice.org.uk/ guidance/cg127. Accessed September 12, 2014.

7. O'Brien E, Parati G, Stergiou G, et al; European Society of Hypertension Working Group on Blood Pressure Monitoring. European Society of Hypertension position paper on ambulatory blood pressure monitoring. J Hypertens. 2013;31(9):1731-1768.

8. Danon-Hersch N, Marques-Vidal P, Bovet P, et al. Prevalence, awareness, treatment and control of high blood pressure in a Swiss city general population: the CoLaus study. Eur J Cardiovasc Prev Rehabil. 2009; 16(1):66-72.
9. Wolf-Maier K, Cooper RS, Kramer H, et al. Hypertension treatment and control in five European countries, Canada, and the United States. Hypertension. 2004;43(1):10-17.

10. Banegas JR, Rodriguez-Artalejo F, de la Cruz Troca JJ, GuallarCastillon P, del Rey Calero J. Blood pressure in Spain: distribution, awareness, control, and benefits of a reduction in average pressure. Hypertension. 1998;32(6):998-1002.

11. Thamm M. [Blood pressure in Germany - current status and trends]. Gesundheitswesen. 1999;61 Spec No:S90-S93. German.

12. Osterberg L, Blaschke T. Adherence to medication. NEngl J Med. 2005; 353(5):487-497.

13. Roumie CL, Elasy TA, Greevy R, et al. Improving blood pressure control through provider education, provider alerts, and patient education: a cluster randomized trial. Ann Intern Med. 2006;145(3): $165-175$.

14. Bobrie G, Postel-Vinay N, Delonca J, Corvol P; SETHI Investigators. Self-measurement and self-titration in hypertension: a pilot telemedicine study. Am J Hypertens. 2007;20(12):1314-1320.

15. Schroeder K, Fahey T, Ebrahim S. Interventions for improving adherence to treatment in patients with high blood pressure in ambulatory settings. Cochrane Database Syst Rev. 2004;2:CD004804.

16. Glynn LG, Murphy AW, Smith SM, Schroeder K, Fahey T. Selfmonitoring and other non-pharmacological interventions to improve the management of hypertension in primary care: a systematic review. Br J Gen Pract. 2010;60(581):e476-e488.

17. Canadian Hypertension Education Program. 2008 Canadian Hypertension Education Program recommendations: an annual update. Can Fam Physician. 2008;54(11):1539-1542.

18. Bender B, Milgrom H, Apter A. Adherence intervention research: what have we learned and what do we do next? J Allergy Clin Immunol. 2003;112(3):489-494.

19. Chmiel C, Wang M, Senn O, et al. Uncontrolled arterial hypertension in primary care - patient characteristics and associated factors. Swiss Med Wkly. 2012;142:w13693.

20. Steurer-Stey C, Zoller M, Chmiel Moshinsky C, Senn O, Rosemann T. Does a colour-coded blood pressure diary improve blood pressure control for patients in general practice: the CoCo trial. Trials. 2010; 11:38.

21. Mansia G, De Backer G, Dominiczak A, et al; European Society of Hypertension; European Society of Cardiology. 2007 ESH-ESC Guidelines for the management of arterial hypertension: the task force for the management of arterial hypertension of the European Society of Hypertension (ESH) and of the European Society of Cardiology (ESC). Blood Press. 2007;16(3):135-232.

22. Mancia G, Fagard R, Narkiewicz K, et al; Task Force Members. $2013 \mathrm{ESH} / \mathrm{ESC}$ Guidelines for the management of arterial hypertension: the Task Force for the management of arterial hypertension of the European Society of Hypertension (ESH) and of the European Society of Cardiology (ESC). J Hypertens. 2013;31(7):1281-1357.

23. Burnier M; 1999 World Health Organization/International Society of Hypertension Guidelines. Blood pressure control and the implementation of guidelines in clinical practice: can we fill the gap? J Hypertens. 2002;20(7):1251-1253.

24. Fahey T, Schroeder K, Ebrahim S. Educational and organisational interventions used to improve the management of hypertension in primary care: a systematic review. Br J Gen Pract. 2005;55(520):875-882.

25. Verberk WJ, Kroon AA, Kessels AG, de Leeuw PW. Home blood pressure measurement: a systematic review. J Am Coll Cardiol. 2005;46(5): $743-751$.

26. Bray EP, Holder R, Mant J, McManus RJ. Does self-monitoring reduce blood pressure? Meta-analysis with meta-regression of randomized controlled trials. Ann Med. 2010;42(5):371-386.

27. Uhlig K, Patel K, Ip S, Kitsios GD, Balk EM. Self-measured blood pressure monitoring in the management of hypertension: a systematic review and meta-analysis. Ann Intern Med. 2013;159(3):185-194.

28. Clark NM, Gong M, Kaciroti N. A model of self-regulation for control of chronic disease. Health Educ Behav. 2001;28(6):769-782. 
29. Bandura A. Self-efficacy: toward a unifying theory of behavioral change. Psychol Rev. 1977;84(2):191-215.

30. Zarnke KB, Feagan BG, Mahon JL, Feldman RD. A randomized study comparing a patient-directed hypertension management strategy with usual office-based care. Am J Hypertens. 1997;10(1):58-67.

31. McManus RJ, Mant J, Bray EP, et al. Telemonitoring and self-management in the control of hypertension (TASMINH2): a randomised controlled trial. Lancet. 2010;376(9736):163-172.

32. Powell H, Gibson PG. Options for self-management education for adults with asthma. Cochrane Database Syst Rev. 2003;1:CD004107.

33. Gibson PG, Powell H. Written action plans for asthma: an evidencebased review of the key components. Thorax. 2004;59(2):94-99.

34. Bodenheimer T, Lorig K, Holman H, Grumbach K. Patient selfmanagement of chronic disease in primary care. JAMA. 2002;288(19): 2469-2475.
35. McAndrew L, Schneider SH, Burns E, Leventhal H. Does patient blood glucose monitoring improve diabetes control? A systematic review of the literature. Diabetes Educ. 2007;33(6):991-1010.

36. Clar C, Barnard K, Cummins E, Royle P, Waugh N; Aberdeen Health Technology Assessment Group. Self-monitoring of blood glucose in type 2 diabetes: systematic review. Health Technol Assess. 2010;14(12): $1-140$.

37. O’Brien E, Atkins N, Stergiou G, et al; Working Group on Blood Pressure Monitoring of the European Society of Hypertension. European Society of Hypertension International Protocol revision 2010 for the validation of blood pressure measuring devices in adults. Blood Press Monit. 2010;15(1):23-38.

\section{Publish your work in this journal}

Patient Preference and Adherence is an international, peer-reviewed, open access journal that focuses on the growing importance of patient preference and adherence throughout the therapeutic continuum. Patient satisfaction, acceptability, quality of life, compliance, persistence and their role in developing new therapeutic modalities and compounds to optimize clinical outcomes for existing disease states are major areas of interest for the journal. This journal has been accepted for indexing on PubMed Central. The manuscript management system is completely online and includes a very quick and fair peer-review system, which is all easy to use. Visit http://www dovepress.com/testimonials.php to read real quotes from published authors. 